\title{
A CONNECTIONIST APPROACH FOR ADAPTIVE LESSON
}

Hassina Seridi-Bouchelaghem, Toufik Sari and Mokthar Sellami

Laboratoire de Recherche informatique (LRI), Badji Mokhtar University, B.P 12 Sidi Amar. Annaba.Algeria.

\begin{abstract}
This paper investigates the use of computational intelligence for adaptive lesson generation in a distance learning environment. A method for adaptive pedagogical hypermedia document generation is proposed and implemented in a prototype called KnowledgeClass. This method is based on specialized connectionist architecture. The domain model is represented in a connectionist-based system which provides an optimal didactic plan composed of a set of basic units. The generated didactic plan is adapted to the learner's goals, abilities and preferences.
\end{abstract}

\section{INTRODUCTION}

Intelligence in a Web-based learning environment (LE) is ensured with tools enabling efficient management of the available information. One of the important keys is the system capability to provide the learner with personalized, adaptive and effective teaching. Thus, our research is focused on the adaptive systems to accommodate diversity in the learner needs, abilities and preferred learning style. Towards this direction, this paper investigates the use of connectionist methods to support the lesson generation process.

For this personalized learning, two models should be built: the domain and the learner model. A connectionist-based structure for representing the domain model is proposed and interactions between the connectionist architecture and the learner model are described. 


\section{THE INTERACTIONS FOR THE GENERATION PROCESS}

The didactic decisions are made at the end of the knowledge evaluation and the identification of the learner preferences and the domain model consultation. The generation of the adaptive didactic plan concretizes the composition of pedagogical documents adapted to the learner profile. The basic idea is to use the learner and the domain models to extract and organize the knowledge in order to satisfy the learning goal. The generation process is carried out in three stages:

- Selection of the learning goal by the learner.

- Planning the content: selection of the suitable concepts for the learning goal.

- Planning the presentation : selection of the basic units hand-annotated and organized in didactic plan for delivering to the learner according to a teaching strategy defined by a learning style.

\section{THE CONNECTIONIST IMPLEMENTATION}

Two Multi-layer Perceptrons (MLP) with one hidden layer are constructed to model the third stage of used information and the decision to make upon learner's understanding.

An artificial neuron calculates a function of all incoming values corresponding to the neurons outputs of the previous layer multiplied by the link's weights. In this neural net each output neuron in the output layer is assigned to a basic unit, while input neurons in the input layer represent the concepts related to the learning goal of the course. The used algorithm for training the MLP is called 'BackPropagation' and it works by calculating the difference between the neural net responses upon input vectors and the desired outputs. The first neural network is used to select the appropriate (UB) for the learner in the first stage of learning. The input layer presented the concepts of the course, one neuron per concept. The input vector (VI) is a $\{-1,1\}$ set where the values $\mathrm{VI}_{\mathrm{i}}=1$ indicates that the corresponding concept $\left(c_{i}\right)$ is important to the learner and the values $V_{i}=-1$ means that $\left(c_{i}\right)$ is not. The (VI) values are set by the evaluation module for the pre-test phase. The second neural network intervenes when the learner don't succeed the posttest of the concepts. This later generates a vector of marks related to concepts called reinforcing vector (VR). The (VR) is used as input values for the second neural network, the output layer for selecting reinforcing documents called (UB). 